\title{
Insulin-sensitive regulation of glucose transport and GLUT4 translocation in skeletal muscle of GLUT1 transgenic mice
}

\author{
Garret J. ETGEN, Jr.*, William J. ZAVADOSKI*, Geoffrey D. HOLMAN $\dagger$ and E. Michael GIBBS ${ }^{\star 1}$ \\ *Pfizer Central Research, Eastern Point Road, Groton, CT 06340, U.S.A., and †Department of Biology and Biochemistry, University of Bath, Claverton Down, \\ Bath BA2 7AY, U.K.
}

\begin{abstract}
Skeletal muscle glucose transport was examined in transgenic mice overexpressing the glucose transporter GLUT1 using both the isolated incubated-muscle preparation and the hind-limb perfusion technique. In the absence of insulin, 2-deoxy-D-glucose uptake was increased $\sim 3-8$-fold in isolated fast-twitch muscles of GLUT1 transgenic mice compared with non-transgenic siblings. Similarly, basal glucose transport activity was increased $\sim 4-14$-fold in perfused fast-twitch muscles of transgenic mice. In non-transgenic mice insulin accelerated glucose transport activity $\sim 2-3$-fold in isolated muscles and to a much greater extent ( $\sim 7-20$-fold) in perfused hind-limb preparations. The observed effect of insulin on glucose transport in transgenic muscle was similarly dependent upon the technique used for measurement, as insulin had no effect on isolated fast-twitch muscle from transgenic mice, but significantly enhanced glucose transport in perfused fast-twitch muscle from transgenic mice to $\sim 50-75 \%$ of the magnitude of the increase observed in nontransgenic mice. Cell-surface glucose transporter content was
\end{abstract}

assessed via 2- $N$-4-(1-azi-2,2,2-trifluoroethyl)benzoyl-1,3-bis-(D mannos-4-yloxy)-2-propylamine photolabelling methodology in both isolated and perfused extensor digitorum longus (EDL). Cell-surface GLUT1 was enhanced by as much as 70-fold in both isolated and perfused EDL of transgenic mice. Insulin did not alter cell-surface GLUT1 in either transgenic or non-transgenic mice. Basal levels of cell-surface GLUT4, measured in either isolated or perfused EDL, were similar in transgenic and nontransgenic mice. Interestingly, insulin enhanced cell-surface GLUT4 2-fold in isolated EDL and 6-fold in perfused EDL of both transgenic and non-transgenic mice. In summary, these results reveal differences between isolated muscle and perfused hind-limb techniques, with the latter method showing a more robust responsiveness to insulin. Furthermore, the results demonstrate that muscle overexpressing GLUT1 has normal insulininduced GLUT4 translocation and the ability to augment glucose-transport activity above the elevated basal rates.

\section{INTRODUCTION}

The facilitated diffusion of glucose across the plasmalemma is considered a rate-limiting step in skeletal muscle carbohydrate metabolism [1], and thus this process represents a key event in the maintenance of whole-body glucose homoeostasis. Glucose transport in skeletal muscle is governed by two isoforms of the facilitated glucose transporter family: GLUT1, which functions primarily as a regulator of basal glucose transport activity, and GLUT4, which responds to various environmental influences altering the rate of glucose transport to meet changing metabolic demands (for review see [1]). In efforts to further our understanding of the regulation of glucose metabolism and in hopes of improving glycaemic control, we and others have developed transgenic mice selectively overexpressing either GLUT1 or GLUT4 [2-10].

Transgenic mice overexpressing GLUT1 exclusively in skeletal muscle exhibit reduced fasting and fed blood-glucose levels and possess an enhanced ability to dispose of a glucose load relative to non-transgenic siblings [3]. These observations are consistent with the finding that basal skeletal muscle glucose-transport activity is significantly enhanced in the GLUT1 transgenic mice relative to controls $[3,4,10]$. Interestingly, however, various stimuli which typically accelerate muscle glucose transport (e.g. insulin, contraction, hypoxia) appear to be without additional effect above the elevated basal level in isolated skeletal muscles of the GLUT1 transgenic mice [10]. It has been proposed that the inability of these stimuli to enhance glucose transport activity in isolated muscles of GLUT1 transgenic mice stems from an inhibition of GLUT4 translocation that is a consequence of the elevated basal glucose transport activity [10]. It has also been postulated that chronically elevated glucose flux may impair GLUT4 translocation via feedback regulation from the hexosamine biosynthetic pathway [11]. Consistent with this hypothesis, it has recently been demonstrated that the activity of glutamine:fructose-6-phosphate amidotransferase (rate-limiting enzyme) and the concentrations of nucleotide-linked sugars were significantly increased in muscles of GLUT1 transgenic mice [12]. Despite these recent observations, the exact mechanism by which elevated glucose flux alters the acute regulation of glucose transport activity in skeletal muscle of GLUT1 transgenic mice has not been demonstrated directly.

To our knowledge, all studies to date examining glucose transport in skeletal muscle of GLUT1 transgenic mice have used isolated muscle preparations $[3,4,10]$. In the present study, initial experiments confirmed that our GLUT1 transgenic mice, which were derived from the originally described transgenic line [3], exhibited the same glucose-transport properties in isolated

Abbreviations used: GLUT1, glucose transporter 1; ATB-BMPA, 2-N-4-(I-azi-2,2,2-trifluoroethyl)benzoyl-1,3-bis-(D-mannos-4-yloxy)-2-propylamine; 2DG, 2-deoxy-D-glucose; EDL, extensor digitorum longus; KHB, Krebs-Henseleit bicarbonate buffer; DATD, N,N'-diallyltartardiamide; TPBS, Triton/PBS.

1 To whom correspondence should be addressed (e-mail gibbsem@pfizer.com). 
skeletal muscle as described in the literature $[3,4,10]$. Importantly, we extend earlier findings through application of the perfused hind-limb preparation to the GLUT1 transgenic mice. In contrast to isolated muscle preparations, this technique utilizes the natural vasculature of the mouse, thereby eliminating any possible confounding limitations due to diffusion, and enabling measurement of glucose transport activity without extensive manipulation of the muscles before the assessment of transport.

In order to examine GLUT4 translocation, or more precisely, cell-surface localization, we utilized the membrane-impermeant glucose transporter photoaffinity reagent 2-N-4-(1-azi-2,2,2trifluoroethyl)benzoyl-1,3-bis-(D-mannos-4-yloxy)-2-

propylamine (ATB-BMPA). Whereas this glucose transporter labelling technique has been employed frequently in skeletal muscle, to our knowledge all previous studies have utilized isolated-muscle preparations [13-17]. As a result of the greater insulin responsiveness we observed in perfused mouse muscle relative to isolated muscle we adapted the ATB-BMPA photolabelling technique for use in the perfused hind-limb preparation as well. As expected, cell-surface GLUT1 was substantially elevated in fast-twitch muscle of GLUT1 transgenic mice relative to control mice. Unexpectedly, however, regardless of whether muscles were photolabelled under isolated or perfused conditions, insulin treatment significantly enhanced cell-surface GLUT4 in muscle of the GLUT1 transgenic mice, which demonstrates that the GLUT4 translocates normally in these animals.

\section{EXPERIMENTAL}

\section{Materials}

ATB-[2- $\left.{ }^{3} \mathrm{H}\right] \mathrm{BMPA}$ was synthesized as described previously [18] and had a specific radioactivity of $10 \mathrm{Ci} / \mathrm{mmol}$. The radiolabelled non-metabolizable glucose analogue 2-[3 $\mathrm{H}]$ deoxy-D-glucose $\left({ }^{3} \mathrm{H}\right.$ 2DG) was purchased from Dupont New England Nuclear (Boston, MA, U.S.A.). Clinical-reagent grade BSA and [U$\left.{ }^{14} \mathrm{C}\right]$ mannitol were purchased from ICN (Costa Mesa, CA, U.S.A.). Insulin (Regular Iletin I) was from Lilly (Indianapolis, IN, U.S.A.). All other reagents were purchased from Sigma.

\section{Transgenic mice}

The construction of GLUT1 transgenic mice on the C57BL/6 $\mathrm{x}$ SJ F2 background has been described previously [3]. Briefly, the minigene contains a $2.47 \mathrm{~kb}$ cDNA fragment encoding the human GLUT1 glucose transporter under the regulation of the $1.2 \mathrm{~kb}$ rat myosin light-chain 2 promoter. Mice used in the current study were obtained by back-crossing the original strain of GLUT1 transgenic mice on the hybrid background with the inbred C57BL/KsJ-Lepr ${ }^{d b} /+$ background. The experiments described below were performed on eighth generation GLUT1 transgenic C57BL/KsJ-Lepr ${ }^{d b} /+$ mice and their non-transgenic siblings; at this stage the mice were $>99 \%$ congenic. All mice used in this study carried only a single copy of the Lepr ${ }^{d b}$ gene, and therefore exhibited a lean, non-diabetic phenotype. The presence of the GLUT1 transgene was assessed by PCR of total genomic DNA isolated from tail snips using the following primer sequences: AAGGGGTCTACATGGCAAC and GCAACGGCTTAGACTTCGACT. All mice were housed in an environmentally controlled animal facility and provided free access to food (Purina chow) and water.

\section{Oral glucose-tolerance test}

Non-anaesthetized GLUT1 transgenic and age-matched nontransgenic siblings $(\sim 30 \mathrm{~g})$ were fasted overnight and bled via the orbital sinus $(0.025 \mathrm{ml})$ immediately before administration of an oral glucose load ( $1 \mathrm{~g}$ of glucose per $\mathrm{kg}$ of body weight) by gavage using a syringe equipped with a murine oral feeding needle (20 gauge; Popper \& Sons, Inc., New Hyde Park, NY, U.S.A.). Mice were subsequently bled after 30, 75 and $120 \mathrm{~min}$. Plasma glucose was measured using the VP Super System Autoanalyzer (Abbot Laboratories, North Chicago, IL, U.S.A.).

\section{Isolated-muscle glucose-transport measurements}

At approx. 11:00h, fed mice ( $\sim 30 \mathrm{~g}$ ) were anaesthetized via an intraperitoneal injection of pentobarbital sodium $(6 \mathrm{mg} / 100 \mathrm{~g}$ of body weight). Epitrochlearis, extensor digitorum longus (EDL) and soleus muscles were surgically isolated, excised and incubated in $1.25 \mathrm{ml}$ of continuously gassed $\left(95 \% \mathrm{O}_{2} / 5 \% \mathrm{CO}_{2}\right) \mathrm{Krebs}-$ Henseleit bicarbonate buffer (KHB) containing $0.1 \%(\mathrm{w} / \mathrm{v})$ BSA $/ 8 \mathrm{mM}$ glucose $/ 32 \mathrm{mM}$ mannitol in either the presence or absence of $13 \mathrm{nM}$ insulin. This insulin concentration maximally stimulated glucose-transport activity in the isolated incubatedmuscle preparation. The initial incubation phase was conducted at $29{ }^{\circ} \mathrm{C}$ for $40 \mathrm{~min}$. Thereafter the muscles were transferred to new vials containing KHB, $0.1 \%$ (w/v) BSA, $40 \mathrm{mM}$ mannitol, in the presence or absence of $13 \mathrm{nM}$ insulin for a $10 \mathrm{~min}$ wash phase at $29^{\circ} \mathrm{C}$. Muscles were then transferred to new vials and glucose-transport activity was measured in KHB containing $0.1 \%$ (w/v) BSA $/ 1 \mathrm{mM} 2 \mathrm{DG}\left(2.25 \mathrm{mCi}\right.$ of $\left.{ }^{3} \mathrm{H}-2 \mathrm{DG} / \mathrm{mmol}\right) /$ $39 \mathrm{mM}$ mannitol $\left(10 \mu \mathrm{Ci}\right.$ of $\left.\left[\mathrm{U}-{ }^{14} \mathrm{C}\right] \mathrm{mannitol} / \mathrm{mmol}\right)$ in the presence or absence of $13 \mathrm{nM}$ insulin at $29^{\circ} \mathrm{C}$ for $20 \mathrm{~min}$. At the end of the final incubation phase muscles were blotted on gauze and clamp-frozen in tongs cooled by liquid nitrogen. Frozen muscles were trimmed of their tendons and weighed before digestion in $0.5 \mathrm{ml}$ of $1 \mathrm{M} \mathrm{KOH}$ at $80{ }^{\circ} \mathrm{C}$ for $30 \mathrm{~min}$. Muscle digests were then neutralized with $0.5 \mathrm{ml}$ of $1 \mathrm{M} \mathrm{HCl}$ and a $0.3 \mathrm{ml}$ aliquot was subjected to dual-label liquid-scintillation spectrophotometry. Glucose-transport activity, expressed in $\mu \mathrm{mol} / 20$ min per $\mathrm{g}$ of muscle, was determined from the intracellular accumulation of ${ }^{3} \mathrm{H}-2 \mathrm{DG}$, as calculated by subtracting extracellular ${ }^{3} \mathrm{H}-2 \mathrm{DG}$ from total muscle ${ }^{3} \mathrm{H}-2 \mathrm{DG}$. Extracellular ${ }^{3} \mathrm{H}-2 \mathrm{DG}$ was estimated from measurement of the extracellular marker $\left[\mathrm{U}-{ }^{14} \mathrm{C}\right]$ mannitol.

\section{Perfused hind-limb glucose-transport measurements}

Between 10:00h and 13:00h, mice $(\sim 30 \mathrm{~g})$ were anaesthetized via an intraperitoneal injection of $1 \mathrm{ml} / \mathrm{kg}$ of Innovar Vet (Pitman Moore, Mundelein, IL, U.S.A.) and $15 \mathrm{mg} / \mathrm{kg}$ diazepam (Hoffman-LaRoche, Nutley, NJ, U.S.A.). With slight modifications, mice were then surgically prepared for hind-limb perfusion as described previously for rats [19]. Briefly, through the placement of ligatures, lower body blood flow was restricted to the right hind limb and 100 units of heparin was administered intravenously to prevent blood clotting. An arterial catheter was then inserted into the abdominal aorta and slid into the right iliac artery, after which a venous catheter was inserted into the abdominal vena cava. The hind limb was then washed out with $5 \mathrm{ml}$ of KHB and during this time a lethal intra-cardiac injection of pentobarbital sodium was administered. The lower hemicorpus was then isolated via transection at the lumbar region and placed in line with a flow-through perfusion system set to deliver continuously gassed $\left(95 \% \mathrm{O}_{2} / 5 \% \mathrm{CO}_{2}\right)$ perfusate at $1 \mathrm{ml} / \mathrm{min}$.

To allow stabilization, hind limbs were perfused for $10 \mathrm{~min}$ with warmed $\left(37^{\circ} \mathrm{C}\right) \mathrm{KHB}$ containing $2.5 \%$ (w/v) BSA $/ 0.2 \mathrm{mM}$ pyruvate $/ 1.0 \mathrm{mM}$ glucose, in either the presence or absence of $100 \mathrm{nM}$ insulin. We found that this insulin concentration maximally stimulated muscle glucose-transport activity and that, in agreement with previous results (for review see [20]), the 
absence of erythrocytes in the perfusate did not significantly alter glucose-transport activity as long as the medium was sufficiently oxygenated (results not shown). After the stabilization phase, glucose transport was measured over a $10 \mathrm{~min}$ period with warmed $\left(37^{\circ} \mathrm{C}\right) \mathrm{KHB}$ containing $2.5 \%$ (w/v) BSA $/ 8 \mathrm{mM} 2 \mathrm{DG}$ $\left(90.6 \mu \mathrm{Ci}\right.$ of $\left.{ }^{3} \mathrm{H}-2 \mathrm{DG} / \mathrm{mmol}\right) / 2 \mathrm{mM}$ mannitol $(120 \mu \mathrm{Ci}$ of $[\mathrm{U}-$ $\left.{ }^{14} \mathrm{C}\right]$ mannitol), in the presence or absence of $100 \mathrm{nM}$ insulin. At the end of the transport phase, the EDL, plantaris and soleus muscles were excised and clamp-frozen in tongs cooled by liquid nitrogen. These muscles were then processed exactly as described above for isolated-muscle glucose-transport measurements, except that glucose transport is expressed in $\mu \mathrm{mol} / \mathrm{h}$ per $\mathrm{g}$ of muscle.

\section{Cell-surface ATB-BMPA-labelling of glucose transporters in isolated muscle}

EDL muscles were isolated and incubated exactly as described above for measurement of glucose-transport activity. At the end of the $10 \mathrm{~min}$ wash phase, however, muscles were transferred to $1 \mathrm{ml}$ of $\mathrm{KHB}$ containing $0.1 \%(\mathrm{w} / \mathrm{v}) \mathrm{BSA} / 1.5 \mathrm{mCi} / \mathrm{ml}$ ATB-[2$\left.{ }^{3} \mathrm{H}\right] \mathrm{BMPA}$, in the presence or absence of $13 \mathrm{nM}$ insulin. After a 5 min incubation, muscles were exposed to $300 \mathrm{~nm}$ UV light in a Rayonet RPR 200 photochemical reactor (Southern New England Ultraviolet Company, Branford, CT, U.S.A,) for $5 \mathrm{~min}$. Muscles were turned over at $2.5 \mathrm{~min}$ to achieve maximum exposure. At the end of the cross-linking step, muscles were frozen in tongs cooled by liquid nitrogen and stored at $-80^{\circ} \mathrm{C}$ until processing.

\section{Cell-surface ATB-BMPA-labelling of glucose transporters in perfused muscle}

Mice ( $\sim 30 \mathrm{~g}$ ) were prepared for hind-limb perfusion exactly as described above for measurement of glucose-transport activity. After the $10 \mathrm{~min}$ stabilization period, however, the perfusate was switched to KHB containing $2.5 \%$ (w/v) BSA/8 mM 2DG/2 $\mathrm{mM}$ mannitol, in the presence or absence of $100 \mathrm{nM}$ insulin, and no radiolabelled sugars. At the end of the $10 \mathrm{~min}$ transport phase, $2 \mathrm{ml}$ of KHB containing $2.5 \%$ (w/v) BSA in the presence or absence of $100 \mathrm{nM}$ insulin was infused to wash out residual extracellular 2DG and mannitol. After the washout, $2 \mathrm{ml}$ of $\mathrm{KHB}$ containing $2.5 \%(\mathrm{w} / \mathrm{v}) \mathrm{BSA}$, in the presence or absence of $100 \mathrm{nM}$ insulin, and ATB-[2- $\left.{ }^{3} \mathrm{H}\right] \mathrm{BMPA}(1.0 \mathrm{mCi} / \mathrm{ml})$ was infused over a 2 min period. After a 5 min equilibration period the skin on the perfused hind limb was pulled back to reveal the musculature and the tibialis anterior was removed, taking care not to disturb the underlying EDL. The hind limb was then pinned at the foot and knee in a specially designed cradle and the entire apparatus was placed in the photochemical reactor for $8 \mathrm{~min}$. At the end of the cross-linking step the EDL was dissected out, frozen in tongs cooled by liquid nitrogen and stored at $-80^{\circ} \mathrm{C}$ until processing.

\section{Processing of cell-surface ATB-BMPA-labelled glucose transporters}

Solubilized crude membranes were prepared from the labelled muscle samples as described previously [14,17]. After sequential immunoprecipitation with affinity-purified rabbit anti-GLUT4 (R1184; specific for the C-terminal 19 amino acids of GLUT4) and affinity-purified goat anti-GLUT1 (G1130; specific for the C-terminal 17 amino acids of GLUT1) at $4{ }^{\circ} \mathrm{C}$, samples were analysed by a modification of the SDS/PAGE procedure described previously [17]. Briefly, $10 \%$ SDS gels were created using the readily soluble cross-linking reagent $N, N^{\prime}$-diallyltartar- diamide (DATD) instead of the customary $N, N^{\prime}$-methylenebisacrylamide. After the samples were run, gels were cut by lane into $4 \mathrm{~mm}$ slices. The substitution of DATD for $N, N^{\prime}-$ methylenebisacrylamide allowed the gel slices to be solubilized immediately without drying at room temperature as a result of the ability of $2 \%$ periodic acid to cleave the cis-diol functional groups on DATD [21]. After a $1 \mathrm{~h}$ solubilization period, scintillation fluid was added and the samples were counted in a liquid-scintillation spectrophotometer. Labelled GLUT4 and GLUT1 for each muscle sample was quantified by integrating the area under the radiolabelled peak and subtracting background radioactivity. Labelled GLUT4 and GLUT1 are expressed in d.p.m./mg wet wt.

\section{Western blot analysis for GLUT4 and GLUT1}

Pooled frozen samples from ten isolated EDL or soleus muscles were homogenized in a 1:20 dilution $(\mathrm{w} / \mathrm{v})$ of sucrose buffer (20 mM Hepes/1 mM EDTA/250 mM sucrose, $\mathrm{pH} 7.4$ ) and aliquots $(50 \mu \mathrm{g}$ of protein) were subjected to SDS/PAGE on a $10 \%$ resolving gel [17]. Resolved proteins were transferred to nitrocellulose membranes, which were blocked in Triton/PBS (TPBS: $0.1 \%$ Triton X-100/10 mM sodium phosphate/150 mM $\mathrm{NaCl}, \mathrm{pH} 7.4$ ) containing $3 \%(\mathrm{w} / \mathrm{v}) \mathrm{BSA}$ (for detection of GLUT1) or $5 \%(\mathrm{w} / \mathrm{v})$ non-fat dry milk (for detection of GLUT4) at room temperature for $1 \mathrm{~h}$, exactly as described previously [22]. Membranes were then incubated overnight with rabbit anti-GLUT1 (R2Ex; raised against purified human erythrocyte GLUT1) antibody or anti-GLUT4 (R1184) antibody at $0.5 \mu \mathrm{g} /$ $\mathrm{ml}$ in TPBS containing $3 \%(\mathrm{w} / \mathrm{v})$ BSA or $1 \%(\mathrm{w} / \mathrm{v})$ non-fat dry milk respectively. GLUT1 and GLUT4 bands were visualized using enhanced chemiluminescence (ECL; Amersham, Corporation, Arlington Heights, IL, U.S.A.) following the manufacturer's instructions.

\section{Statistical analysis}

All results are expressed as means \pm S.E.M.. The data were analysed via analysis of variance with significant differences between means identified using Fisher's protected least-squares difference post hoc analysis. Significance was set at the $P<0.05$ level.

\section{RESULTS}

\section{Western blot analysis and oral glucose-tolerance test}

Since the mice used in this study were eight generations removed from the original GLUT1 transgenic mouse line that has been extensively characterized $[3,4,10]$, it was first necessary to confirm that expression of the GLUT1 transgene was maintained and that a similar phenotype of enhanced glucose disposal was observed. Western blot analysis (results not shown) revealed marked overexpression of GLUT1 in fast-twitch EDL muscle, as was shown with the original line of transgenic mice $[3,4]$. Furthermore, the level of overexpression of the GLUT1 transgene is much less pronounced in the predominantly slow-twitch soleus muscle. GLUT4 levels were not different between transgenic and non-transgenic mice in EDL or soleus muscles (results not shown).

Enhanced glucose disposal in vivo is clearly demonstrated in oral glucose-tolerance tests in which fasting blood glucose was reduced and the sugar load disposed of more rapidly in transgenic than non-transgenic mice (Figure 1). 


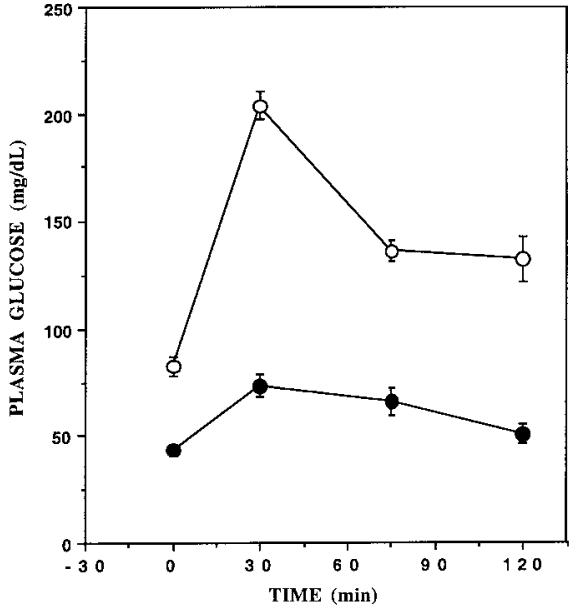

Figure 1 Non-transgenic and transgenic mice were fasted overnight and then given $1 \mathrm{~g}$ of glucose $/ \mathrm{kg}$ of body weight by oral gavage

Plasma glucose levels were determined at the times indicated. Values are expressed as means \pm S.E.M. from 10 non-transgenic $(O)$ and 11 transgenic $(\mathcal{O})$ mice. All values for transgenic mice were significantly different from non-transgenic mice $(P<0.05)$.

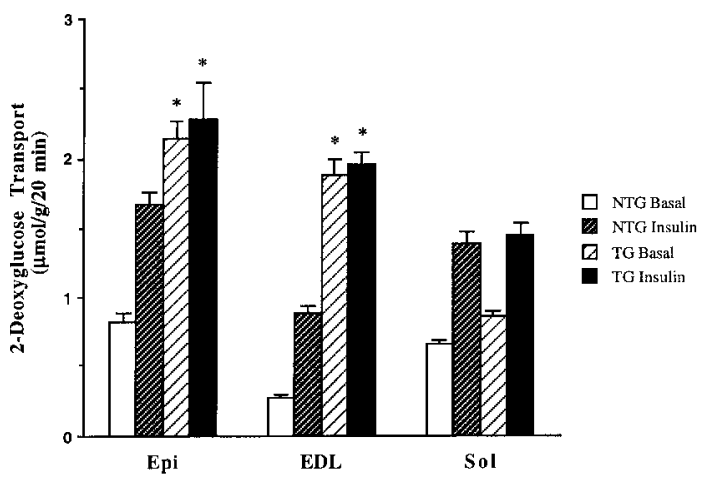

Figure 2 Basal and insulin-stimulated (13 nM) 2DG-transport activity in isolated epitrochlearis (Epi), EDL and soleus (Sol) muscles from nontransgenic (NTG) and GLUT1 transgenic (TG) mice

${ }^{*}$ Significantly different from correspondingly treated non-transgenic muscle, $P<0.05$.

\section{Isolated muscle glucose transport activity}

Glucose-transport activities in isolated epitrochlearis, EDL and soleus muscles from transgenic and non-transgenic mice are displayed in Figure 2. In the absence of insulin glucose transport was elevated $\sim 3$-fold in epitrochlearis and $\sim 7$ fold in EDL isolated from transgenic mice compared with controls, but was not significantly elevated in soleus from transgenic mice. Addition of insulin to the incubation medium enhanced glucose transport activity $\sim 2-3$-fold above basal levels in epitrochlearis and EDL from non-transgenic mice and $\sim 2$-fold in soleus from both nontransgenic and transgenic mice. In contrast, insulin had little effect above the high basal levels on glucose-transport activity in epitrochlearis or EDL from transgenic mice, in agreement with previously published results [10].

\section{Perfused-muscle glucose-transport activity}

Glucose-transport activities in perfused plantaris, EDL and soleus muscles from transgenic and non-transgenic mice are

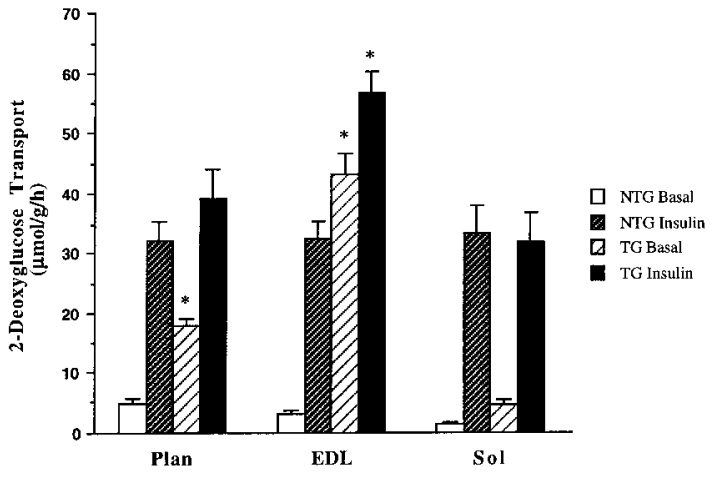

Figure 3 Basal and insulin-stimulated (100 nM) 2DG-transport activity in perfused, plantaris (Plan), EDL and soleus (Sol) muscles from non-transgenic (NTG) and GLUT1 transgenic (TG) mice

*Significantly different from correspondingly treated non-transgenic muscle, $P<0.05$.

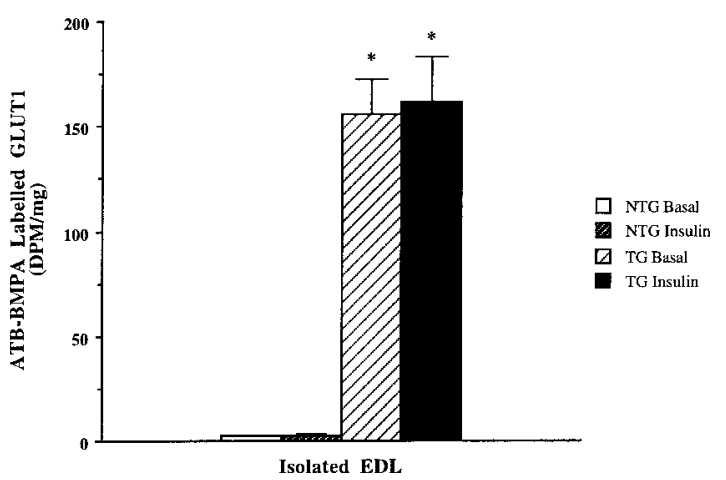

Figure 4 ATB-BMPA-labelled cell-surface GLUT1 in basal and insulintreated (13 nM) isolated EDL from non-transgenic (NTG) and GLUT1 transgenic (TG) mice

*Significantly different from correspondingly treated non-transgenic muscle, $P<0.05$

displayed in Figure 3. Similar to the results in isolated muscle, in the absence of insulin glucose transport was elevated $\sim 4$-fold in plantaris and $\sim 13$-fold in EDL from transgenic mice compared with controls, but was not significantly altered in soleus from transgenic mice. The presence of insulin in the perfusate enhanced glucose transport $\sim 7-20$-fold above basal levels in muscles from non-transgenic mice and $\sim 7$-fold above basal levels in soleus from transgenic mice. Surprisingly, despite the substantial elevation of basal transport rates, insulin significantly $(P<0.05)$ enhanced glucose transport in perfused plantaris and EDL from transgenic mice to $50-75 \%$ of the increment observed in non-transgenic muscles.

\section{Cell-surface ATB-BMPA labelling of glucose transporters in isolated muscle}

Cell-surface GLUT1 in isolated EDL from transgenic and nontransgenic mice is presented in Figure 4. Astonishingly, in transgenic EDL the level of GLUT1 at the cell surface was elevated $\sim 70$-fold over non-transgenic levels. In neither transgenic nor non-transgenic EDL did cell-surface GLUT1 levels change in the presence of insulin. 


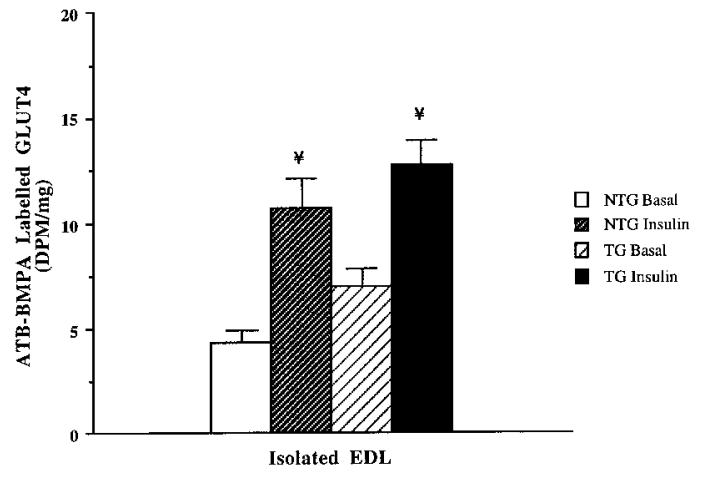

Figure 5 ATB-BMPA-labelled cell-surface GLUT4 in basal and insulintreated (13 nM) isolated EDL from non-transgenic (NTG) and GLUT1 transgenic (TG) mice

YSignificantly different from basal, $P<0.05$.

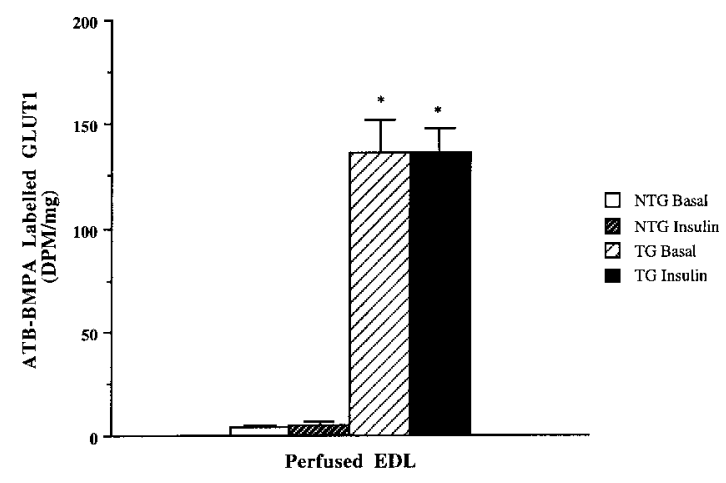

Figure 6 ATB-BMPA-labelled cell-surface GLUT1 in basal and insulin (100 nM)-perfused EDL from non-transgenic (NTG) and GLUT1 transgenic (TG) mice

${ }^{*}$ Significantly different from correspondingly treated non-transgenic muscle, $P<0.05$.

Cell-surface GLUT4 in isolated EDL from transgenic and non-transgenic mice is presented in Figure 5. Basal cell-surface GLUT4 levels were the same in transgenic and non-transgenic EDL. As anticipated, insulin increased cell-surface GLUT4 in non-transgenic EDL 2-fold, however, unexpectedly, a similar insulin-stimulated increase in cell-surface GLUT4 ( $\sim 2$-fold) was observed in transgenic EDL.

\section{Cell-surface ATB-BMPA labelling of glucose transporters in perfused muscle}

Cell-surface GLUT1 and GLUT4 in EDL from transgenic and non-transgenic mice measured using the novel ATB-BMPA perfusion technique are presented in Figures 6 and 7. Similar to results from isolated muscle, cell-surface GLUT1 in perfused muscle was substantially elevated in EDL of transgenic mice $(\sim$ 40-fold over non-transgenic levels). Insulin had no effect on cellsurface GLUT1 in either transgenic or non-transgenic perfused EDL. On the other hand, insulin clearly enhanced cell-surface GLUT4 in both non-transgenic and transgenic perfused EDL, and to a greater extent than observed in isolated EDL (5-6-fold versus $\sim 2$-fold respectively).

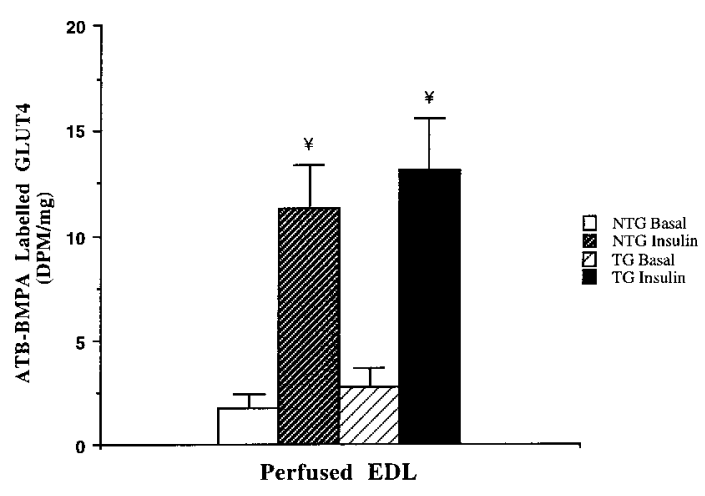

Figure 7 ATB-BMPA-labelled cell-surface GLUT4 in basal and insulin (2500 nM)-perfused EDL from non-transgenic (NTG) and GLUT1 transgenic (TG) mice

YSignificantly different from basal, $P<0.05$.

\section{DISCUSSION}

Preliminary phenotyping of the mice used in this study demonstrates that GLUT1 overexpression and its physiological actions were maintained during the extensive back-crossing needed for the production of the mice used in the present study; the results support the concept that elevated basal glucose transport in skeletal muscle can alter whole-body glucose homoeostasis. A confounding repercussion of the elevated glucose flux in GLUT1 transgenic mice that has been reported is the absence of insulin responsiveness in isolated skeletal muscle [10], and decreased insulin sensitivity in euglycaemic clamps [23]. In the present investigation we sought to determine, at the level of the glucose transporters, the mechanism through which GLUT1-overexpressing muscles apparently lose the ability to respond markedly to an insulin stimulus.

Western blot analysis demonstrated that GLUT1 overexpression did not alter skeletal muscle GLUT4 levels. These results confirm previous observations [3] and demonstrate that the apparent lack of insulin responsiveness in isolated skeletal muscle from GLUT1 transgenic muscle is not due to GLUT4 down regulation.

Our results support previous observations $[6,7,10]$ demonstrating that glucose-transport activity in mouse muscle, measured under isolated incubated conditions, exhibits relatively low insulin responsiveness $(\sim 2-3$-fold increase above basal glucose transport). A possible reason for the low insulin responsiveness seen in soleus and EDL may be an elevation of basal glucose transport due to compromised oxygen delivery to deeper muscle fibres, as the thickness of these muscles is near the threshold at which hypoxia is predicted to occur in rat muscles [20]. Our epitrochlearis results, however, are not consistent with this hypothesis, as we have observed murine epitrochlearis to be only 8-12 fibers thick, which should not be limited by diffusion, yet, similar to the soleus and EDL, insulin enhanced glucose transport only 2-fold above basal levels. Another possible reason why basal glucose-transport rates may be elevated in the isolatedmuscle preparation stems from the unavoidable mechanical manipulation of the muscle during the excision process.

In contrast to isolated-muscle preparations, however, Brozinick et al. [9] recently demonstrated, using the hind-limb perfusion technique, that pooled muscle from mouse hind limb may be more insulin responsive than previously suggested from use of other techniques. Therefore, we examined glucose 
transport using the hind-limb perfusion technique in an attempt to assess more fully insulin responsiveness in GLUT1 transgenic muscle. Insulin accelerated glucose transport up to 20 -fold above basal levels in non-transgenic muscle, as assessed using the hindlimb perfusion technique. These findings clearly demonstrate that, under appropriate conditions, mouse muscle can be substantially more insulin responsive than indicated by results from the isolated skeletal muscle preparations. A similar conclusion has been alluded to previously in response to glucose-utilization measurements during euglycaemic clamps [23].

Aside from the observation of technique-dependent differences in murine muscle insulin responsiveness, a major finding from our hind-limb perfusion experiments was that in contrast to results from isolated muscle, perfused fast-twitch transgenic muscles were able to respond to insulin stimulation, as evidenced by a further acceleration of glucose transport despite elevated basal glucose flux. This finding not only contradicts the conclusion concerning lack of insulin sensitivity reached from isolated muscle experiments performed by us (Figure 2) and others $[3,4,10]$, but also provides the first indication that the hypothesis that insulin could not stimulate GLUT4 translocation in GLUT1 transgenic mouse muscle is incorrect. In order to address this issue, we employed the impermeant photoaffinity reagent ATB-BMPA to directly quantify cell-surface glucose transporter levels.

Photoaffinity labelling of glucose transporters was performed in isolated EDL in a manner similar to that described previously [17]. However, because of the enhanced insulin responsiveness in both non-transgenic and transgenic mice observed in the perfused muscle, we also developed a novel technique for photolabelling the perfused EDL. The EDL was chosen for study because of the high GLUT1 expression levels of this muscle type in the transgenic mice and the ease with which it may be separated from surrounding tissues in situ. Regardless of whether transporters were photolabelled in the traditional incubated isolated-muscle preparation or in the newly adapted perfused hind-limb preparation the results were qualitatively similar. Strikingly, cellsurface GLUT1 in the transgenic EDL was elevated an average of 70- or 40-fold over levels found in the non-transgenic EDL when assessed using the isolated or perfused hind-limb preparations respectively. Cell-surface GLUT1 did not change with insulin stimulation in EDL when assessed with either photolabelling method. These results clearly support the role of GLUT1 in basal muscle glucose transport and demonstrate the mechanism through which basal glucose-transport activity is elevated in muscle of GLUT1 transgenic mice.

Unexpectedly, regardless of whether assessed using the isolated-muscle or perfused hind-limb methodology, cell-surface GLUT4 increased significantly in response to insulin in the EDL of both transgenic and non-transgenic mice. With regard to the non-transgenic mice, the changes in cell-surface GLUT4 closely correspond with the changes seen in glucose transport for both isolated and perfused conditions. These results are similar to previous reports from isolated rat skeletal muscle and support the hypothesis that in normal muscle insulin exerts its effect on glucose transport mainly through changes in surface-accessible GLUT4 $[14,17]$. Although insulin did not markedly alter glucosetransport activity in isolated transgenic EDL above the high basal levels, cell-surface GLUT4 increased 2-fold under these conditions. In perfused transgenic EDL, cell-surface GLUT4 increased $\sim 4$.7-fold.

Whereas the contribution of GLUT1 to transport activity in non-transgenic muscle is nearly negligible, accounting for the contribution of GLUT1 in muscles which highly express this isoform appears to be of paramount importance in establishing a meaningful relationship between transport and transporters. In the transgenic animals, the 40-70-fold increase in GLUT1 increases transport rates in both basal and insulin-stimulated states to such an extent that the expected fold change in transport (above the elevated basal levels) due to translocation of GLUT4 is small. Nevertheless, using the perfusion technique we have found evidence for such an increase. In both transgenic and nontransgenic animals, the correlation between the sum of the GLUT1 and GLUT4 levels and the glucose-transport activity is strong if GLUT1 has approximately half the catalytic activity of GLUT4 [24]. However, the transport increase in transgenic animals is approx. $50-70 \%$ of the increase in non-transgenic animals. This relatively small discrepancy may be related to inaccuracies in measuring the elevated transport rates. Alternatively it may represent a glucose toxicity effect on transport, but not GLUT4 translocation (as previously suggested), arising indirectly from the elevated flux through GLUT1 [11,12,25]

In summary, the results of the present study have several important implications, including the notable revelation that mouse skeletal muscle is substantially more insulin responsive than suggested from isolated-muscle experiments. The major finding of this study, however, is that skeletal muscle of GLUT1 transgenic mice demonstrates completely normal insulinstimulated GLUT4 translocation as measured in both isolated and perfused EDL. This ability of insulin to stimulate GLUT4 translocation in muscle of GLUT1 transgenic mice translates into elevation of glucose transport activity in perfused muscle, despite the considerable elevation of basal glucose transport.

We thank Scott C. McCoid and Paul D. McGill for establishing the GLUT1 transgenic mice on the $>99 \%$ congenic C57BL/KsJ-Lepr ${ }^{d b} /+$ background. We thank the Medical Research Council (U.K.) for support for G.D.H.'s laboratory.

\section{REFERENCES}

1 Mueckler, M. (1994) Eur. J. Biochem. 219, 713-725

2 Ren, J. M., Marshall, B. A., Gulve, E. A., Gao, J., Johnson, D. W., Holloszy, J. 0. and Mueckler, M. (1993) J. Biol. Chem. 268, 16113-16115

3 Marshall, B. A., Ren, J. M., Johnson, D. W., Gibbs, E. M., Lillquist, J. S., Soeller, W. C., Holloszy, J. O. and Mueckler, M. (1993) J. Biol. Chem. 268, 18442-18445

4 Ren, J. M., Marshall, B. A., Gulve, E. A, Gao, J., Johnson, D. W., Holloszy, J. O. and Mueckler, M. (1993) J. Biol. Chem. 268, 16113-16115

5 Liu, M. L., Gibbs, E. M., McCoid, S. C., Milici, A. J., Stukenbrok, H. A., McPherson, R. K., Treadway, J. L. and Pessin, J. E. (1993) Proc. Natl. Acad. Sci. U.S.A. 90, 11346-11350

6 Hansen, P. A., Gulve, E. A., Marshall, B. A., Gao, J., Pessin, J. E., Holloszy, J. 0. and Mueckler, M. (1994) J. Biol. Chem. 269, 1679-1684

7 Treadway, J. L., Hargrove, D. M., Nardone, N. A., McPherson, R. K., Russo, J. F. Milici, A. J., Stukenbrok, H. A., Gibbs, E. M., Stevenson, R. W. and Pessin, J.E (1994) J. Biol. Chem. 269, 29956-29961

8 Gibbs, E. M., Stock, J. L., McCoid, S. C., Stukenbrok, H. A., Pessin, J. E., Stevenson, R. W., Milici, A. J. and McNeish, J. D. (1995) J. Clin. Invest. 95, 1512-1518

9 Brozinick, Jr., J. T., Yaspelkis, III, B. B., Wilson, C. M., Grant, K. E., Gibbs, E. M., Cushman, S. W. and Ivy, J. L. (1996) Biochem. J. 313, 133-140

10 Gulve, E. A., Ren, J. M., Marshall, B. A., Gao, J., Hansen, P. A., Holloszy, J. O. and Mueckler, M. (1994) J. Biol. Chem. 269, 18366-18370

11 Robinson, K. A., Sens, D. A. and Buse, M. G. (1993) Diabetes 42, 1333-1346

12 Buse, M. G., Robinson, K. A., Marshall, B. A. and Mueckler, M. (1996) J. Biol Chem. 271, 23197-23202

13 Lund, S., Holman, G. D., Schmitz, O. and Pedersen, 0. (1993) FEBS Lett. 330, 312-318

14 Wilson, C. M. and Cushman, S. W. (1994) Biochem. J. 299, 755-759

15 Lund, S., Flyvbjerg, A., Holman, G. D., Larsen, F. S., Pedersen, 0. and Schmitz, 0. (1994) Am. J. Physiol. 267, E461-E466

16 Lund, S., Holman, G. D., Schmitz, O. and Pedersen, 0. (1995) Proc. Natl. Acad Sci. U.S.A. 92, 5817-5821

17 Etgen, Jr., G. J., Wilson, C. M., Jensen, J., Cushman, S. W. and Ivy, J. L. (1996) Am. J. Physiol. 271, E294-E301

18 Clark, A. E. and Holman, G. D. (1990) Biochem. J. 269, 615-622 
19 Ruderman, N. B., Houghton, R. S. and Hems, R. (1971) Biochem. J. 124, 639-645

20 Bonen, A., Clark, M. G. and Henriksen, E. J. (1994) Am. J. Physiol. 266, E1-E16

21 Spath, P. J. and Koblet, H. (1979) Anal. Biochem. 93, 275-285

22 Brant, A. M., McCoid, S. C., Thomas, H. M., Baldwin, S. A., Davies, A., Parker, J. C., Gibbs, E. M. and Gould, G. W. (1992) Cell. Signalling 4, 641-650

Received 8 May 1998/25 September 1998; accepted 14 October 1998
23 Marshall, B. A. and Mueckler, M. M. (1994) Am. J. Physiol. 267, E738-E744

24 Palfreyman, R. W., Clark, A. E., Denton, R. M., Holman, G. D. and Kozka, I. J. (1993) Biochem. J. 284, 275-282

25 Garvey, W. T., Olefsky, J. M., Matthei, S. and Marshall, S. (1987) J. Biol. Chem 262, 189-197 